\title{
Coulisses
}

Revue de théâtre

44 | Printemps 2012

Le Hors-scène

\section{Le récit du Mendiant : une approche du « hors- scène » dans Électre de Giraudoux}

Souad Zaied Akrout

\section{(2) OpenEdition}

1 Journals

Édition électronique

URL : https://journals.openedition.org/coulisses/462

DOI : 10.4000/coulisses.462

ISSN : 2546-9460

Éditeur

Presses universitaires de Franche-Comté

Édition imprimée

Date de publication : 1 juin 2012

Pagination : 77-83

ISBN : 978-2-84867-425-4

ISSN : $1150-594 X$

Référence électronique

Souad Zaied Akrout, « Le récit du Mendiant : une approche du «hors-scène » dans Électre de Giraudoux », Coulisses [En ligne], 44 | Printemps 2012, mis en ligne le 30 novembre 2016, consulté le

29 décembre 2022. URL : http://journals.openedition.org/coulisses/462 ; DOI : https://doi.org/

$10.4000 /$ coulisses.462

Tous droits réservés 


\section{Le récit du Mendiant : une approche du « hors-scène » dans Électre de Giraudoux}

SOUAD ZAIED AKROUT

Université Paris 3 - Sorbonne Nouvelle

La scène 9 de l'acte II d'Électre ${ }^{1}$ a cette particularité de s'articuler toute entière autour des deux récits du mendiant: le meurtre d'Agamemnon et les meurtres de Clytemnestre et d'Égisthe. Le premier récit révèle ce qui s'est passé "voilà sept ans »: de retour de Troie, Agamemnon est assassiné par sa femme Clytemnestre et son amant Égisthe dans son palais d'Argos. De ce temps hors de la scène ou de cette scène hors du temps, la vérité vient enfin à Électre. Oreste au cri de sa sœur sort de scène pour assouvir une vengeance qui désormais est sienne. Mais avant de quitter la scène, il lance au mendiant: «Pourquoi t'interrompre, mendiant? Continue. Raconte-leur la mort de Clytemnestre et d'Égisthe!» Ainsi se met en scène, si l'on ose dire, le horsscène le plus célèbre du théâtre giralducien. Nous aborderons ce récit en interrogeant deux notions dramaturgiques fondamentales : l'espace et le temps.

D'abord l'espace: matériel, concret mais aussi psychologique, invisible, mythique. Ensuite le temps, celui du récit et celui de l'action. Nous nous intéresserons particulièrement aux temps verbaux et à ce que Chevrel $^{2}$ intitule «la mise en fiction du temps». Enfin nous essayerons de montrer comment la mise en scène de Jouvet a, dans ce cas précis, représenté le hors-scène.

1. Jean Giraudoux, Électre, Théatre complet, Paris, Gallimard, "Bibliothèque de la Pléiade », édition publiée sous la direction de Jacques Body, 1982.

2. Y Yves Chevrel, " "J'ai raconté trop vite il me rattrape." Quelques remarques sur la mise en fiction du temps dans l'Électre de Giraudoux », Sur une note juste, Mélanges offerts à Jacques Body, réunis par P. Citti, M. Détrie, G. Tessier, Université de Tours, 1990, p. 89-95. 


\section{L'espace}

Le décor du deuxième acte est une "Cour intérieure dans le palais d'Agamemnon ». l'espace de la scène est délimité, encadré, clos. À la question d'Oreste qui veut savoir où trouver les régicides, la Femme Narsès répond : "Dans la cour du sud». Le hors-scène est donc d'emblée défini. Oreste quitte la scène pour venger son père et c'est désormais dans le « hors-scène » que s'accomplit son destin. De ce hors-scène, nous ne savons pas grand-chose, un détail, « une niche en marbre » depuis laquelle est penché Égisthe, écho de l'espace du meurtre. Au-delà d'un premier hors-scène (encore à l'intérieur du palais), il en est un autre : Argos, pour qui Égisthe s'est pris d'amour. D'ailleurs cette niche d'où il se penche est un espace intermédiaire à la fois espace du crime et espace de la rédemption. La niche, synecdoque du palais des Atrides, symbolise l'espace du crime. Elle donne aussi sur Argos qui s'est offerte à Égisthe en un espoir ultime de rédemption. Mais Clytemnestre fait bien vite basculer les choses. En s'accrochant à lui, elle le ramène pour toujours dans le palais des Atrides. Le mythe peut reprendre là où il s'est arrêté. Égisthe mourra en infâme.

Dans la tradition théâtrale, le hors-scène prend à sa charge ce qui ne sied pas à la représentation. Le récit du Mendiant, s'il semble de prime abord obéir à cette règle de bienséance, ne fait en réalité que renforcer davantage l'illusion que veut créer Giraudoux. Électre est une pièce qui semble en tout point se conformer aux codes classiques: unité de temps (la pièce aura lieu en douze heures), de lieu (le palais d'Agamemnon), et d'action. Giraudoux dans une interview précise encore: "j’ai tenté de refaire une place à deux modes d'expression que j'aime beaucoup chez les auteurs anciens : le monologue et le récit $»^{3}$. Pourtant ce n'est qu'une façade. Si le meurtre se passe hors de la vue du spectateur, c'est que le hors-scène n'est pas seulement un espace matériel, concret mais surtout un espace psychologique, un espace qui se construit à travers la perception des événements propre à chaque personnage.

Le Mendiant (et les mendiants ne sont-ils pas souvent des dieux déguisés ?) est omniscient; au-delà du hors-scène, c'est l'espace propre de chacun des personnages qu'il met à nu. Le hors-scène devient dès lors une multitude d'espaces propres, d'espaces psychologiques. D'abord, celui d'Égisthe «qui entendit crier dans son dos une bête qu'on saignait». Le récit du Mendiant prend ainsi à sa charge de révéler les pensées non formulées du personnage, son exaspération (" Il la secouait pour reprendre son bras. Rien à faire. »; «Il y avait encore cet oiseau qui le giflait de ses ailes et l'attaquait du bec $»)$, son désespoir (" désespéré de mourir en criminel quand tout lui était devenu pur et sacré, de combattre pour un cri qui n'était plus le sien [...] de se trouver l'infâme en face de ce parricide, il lutta de sa main que l'épée découpait peu à

3. Interview donnée à Yvonne Moustiers, L'Intransigeant, 30 avril 1937. 
peu »), ses désirs (« et l'on sentait que s’il voulait maintenant se débarrasser de la reine, ce n'était plus pour combattre seul mais pour mourir seul...»). Ensuite celui d'Oreste qui « avait frappé au hasard sur le couple en fermant les yeux » et qui «avait l'impression que c'était une autre mère, une mère innocente qu'il tuait. » Le récit ne se contente pas de raconter au spectateur des faits, il les fait revivre à travers les différentes perspectives des personnages. Le hors-scène n'est plus envisagé comme un espace tridimensionnel invisible mais comme un espace de l'omniscience.

Dans Électre, le hors-scène est aussi l'espace du mythe. C'est derrière la façade du palais que s'est mise en place la malédiction des Atrides. Ainsi, nous savons dès la scène liminaire que la fenêtre aux jasmins est celle où «Atrée, le premier roi d'Argos, tua les fils de son frère », que «le repas où il servit leurs cœurs eut lieu dans la salle voisine», que "Cassandre fut étranglée dans l'échauguette » et que la fenêtre avec les roses est celle de la " piscine » où le roi Agamemnon trouva la mort. C'est donc naturellement derrière cette façade, dans la cour sud du palais, à côté de la niche en marbre d'Argos, que le dernier volet de la malédiction devra avoir lieu. Le meurtre de Clytemnestre et d'Égisthe n'a pas seulement lieu dans le hors-scène, il a lieu dans l'espace du mythe. En effet, nous avons tous les éléments de l'histoire en tête: Oreste venge son père en tuant Clytemnestre et Égisthe. Mais dans le mythe giralducien, Oreste frappe au hasard, en fermant les yeux et Égisthe, auquel on a offert Argos un matin de lumière, ne veut pas mourir en criminel ; mais la légende se referme sur lui avec ce vautour qui descend du ciel pour joindre ses coups à ceux d'Oreste et qui semble, selon Jacques Body, être un signe de «la vengeance des dieux $»^{4}$. Son cri final «Électre» nous parvient non seulement du hors-scène mais peut-être d'un hors mythe, où il aurait pu y avoir un couple Égisthe / Électre. Et nous ne pouvons nous empêcher de penser à la fin d'Ondine : «Comme c'est dommage ! Comme je l'aurais aimé ! » Le hors-scène serait dès lors un espace double, à la fois partie intégrante de l'espace dramatique et espace de tous les possibles. Les lois scéniques ne s’y appliquent pas de la même manière, le temps n'y suit pas le même cours.

\section{Le temps}

L'écriture du temps dans ce passage d'Électre a fait couler beaucoup d'encre et n'a de cesse de nous plonger, comme le dit Jacques Body, dans « des abîmes de perplexité $»^{5}$. En effet, le récit du Mendiant soulève plusieurs interrogations auxquelles nous essaierons d'apporter une réponse. Le récit est d'abord un récit

\footnotetext{
4. Jacques Body, "Légende et dramaturgie dans le théâtre de Giraudoux », Revue d'bistoire littéraire de la France, novembre 1977, nº ${ }^{\circ}$, p. 937.

5. Ibid.
} 
«per eventum» et non pas «post eventum» comme le précise Jacques Body. En effet, comme nous allons le voir, le Mendiant semble nous rapporter les événements au moment même de leur déroulement, allant même jusqu'à « raconter trop vite », devançant ainsi les événements. Il termine son récit alors que le mythe se joue encore en coulisse.

Pourtant le récit du Mendiant commence bien après la sortie d'Oreste (18 répliques plus tard). Nous avons ainsi des indicateurs temporels : à la Femme Narsès qui lui demande de raconter, le Mendiant répond: " Deux minutes. Laisse-lui le temps d'arriver». Un peu plus loin nous avons : «une minute. Voilà il les rejoint. » On s'attendrait après cette réplique à ce que le récit commence, mais ce n'est pas encore le cas. Quatre répliques plus loin, le Mendiant commence enfin à raconter, mais là encore le spectateur est surpris par un autre décalage temporel puisque le récit débute non pas au moment où Oreste arrive devant Égisthe et Clytemnestre mais avant: "Alors voici la fin. La femme Narsès et les mendiants délièrent Oreste. Il se précipita à travers la cour.» De plus, avant que le Mendiant n'entame son récit, Électre a entendu un cri ("on a crié») puis un deuxième ("on a crié cette fois»), un cri qui correspondrait peut-être à celui de Clytemnestre (« et ce n'était pas une bête qui a crié mais Clytemnestre »). Si nous tenons compte de tous ces éléments le récit devrait non pas commenter les événements mais les rapporter, le meurtre serait dès lors antérieur au récit ou du moins simultané. Les événements racontés devraient trouver leur aboutissement sur scène et non pas l'inverse. La logique aurait voulu que le cri d'Égisthe nous parvienne avant la fin du récit du Mendiant et non pas après: "Mais il est mort en criant un nom que je ne dirai pas. / LA VOIX D'ÉGISTHE, au dehors: Électre! » Giraudoux semble s'amuser à brouiller les pistes, ou essaye simplement de nous révéler un secret : le temps au théâtre n'est peut-être pas toujours rectiligne.

D'ailleurs, intéressons-nous de plus près à la célèbre réplique paradoxale du Mendiant: "J'ai parlé trop vite. Il me rattrape.» En effet, comme le dit Chevrel : «dans un emploi usuel du langage, le fait d'aller trop vite [...] implique plutôt que, loin, d'être rattrapé, on a quelque chance de distancer quelqu'un. » Jacques Body, quant à lui, affirme : «Entre le temps du drame et celui de la légende, les rapports sont si confus qu'ils s'inversent aisément comme rattrape, finalement, le montre bien.» Cette fissure qui se crée entre deux temporalités parallèles crée un effet de surprise dramaturgique, le horsscène finit par s'inviter sur scène avec ce cri qui retentit, ce nom que le Mendiant - et peut être la légende - voulait taire et qui nous rattrape. Dès lors ce 
pronom « il » n'est-il pas, comme dans la scène liminaire de La Guerre de Troie n'aura pas lieu, une forme du destin ${ }^{6}$ ?

Oreste quitte la scène en demandant au Mendiant de raconter le meurtre d'Égisthe et de Clytemnestre. Le récit du Mendiant, laissant à Oreste le temps d'arriver à destination, commence à un moment antérieur de l'action avec des verbes au passé simple (« délièrent Oreste, il se précipita, il ne toucha pas, il n’embrassa même pas ») dont la fonction est, comme le souligne Larthomas ${ }^{7}$, d'en «marquer les étapes de la progression ». Soudain, un passé composé ( il a eu tort») vient s'interposer et le temps semble tout à coup se figer. Nous ne sommes plus dans le récit, nous sommes dans le mythe et voilà le Mendiant / Oracle qui nous annonce : «il ne la touchera jamais plus ». La force inéluctable du futur, doublée de cette négation absolue, rappelle au spectateur que le hors-scène n'est pas seulement l'ici / maintenant de la représentation mais aussi et surtout un espace hors du temps, un espace où Oreste va à la rencontre de son destin. Le récit reprend au passé simple (" il atteignit les assassins ») puis nous retrouvons des verbes à l'imparfait (" parlementaient, disait, criait, tuait...»). L'action s'étend, s'épaissit, se répète, le temps prend consistance, le récit s'étoffe, puis le passé simple revient. C'est la fin du récit qui approche, le temps s'accélère, les verbes se succèdent : «Il lutta de sa main que l'épée découpait peu à peu mais le lacet de sa cuirasse se prit dans une agrafe de Clytemnestre et elle s'ouvrit. Alors il ne résista plus [...]». Deux verbes au passé composé - temps du passé « acquis, irrévocable » comme le dit Larthomas ${ }^{8}-$ encadrent la fin du récit : «il n’y est pas parvenu / il est mort». Ces verbes scellent à jamais le mythe d'Électre comme le confirme l'utilisation du présent : « il y a pour toujours un couple Clytemnestre-Égisthe. » Le futur qui vient s'ajouter à cette palette de temps verbaux ("je ne dirai pas») semble ancrer le mythe non pas dans un temps, mais dans le Temps, Passé, Présent, Futur: dans le hors-scène, la boucle est bouclée.

\section{La mise en scène}

Le hors-scène, comme nous avons essayé de le montrer, est multiple, protéiforme. Il est à la fois un prolongement de la scène et un espace-temps indéfinissable et autonome, d'où la difficulté d'essayer de le cerner, de le délimiter, de l'expliquer mais surtout de le représenter. À la création d'Électre, le 13 mai 1937 au théâtre de l'Athénée, Louis Jouvet est confronté à cette difficulté. Nous avons essayé de reconstituer ce parcours grâce notamment aux témoignages et

6. «CASSANDRE: Et il monte sans bruits les escaliers du palais. Il pousse du mufle les portes... Le voilà... Le voilà... » (Jean Giraudoux, La Guerre de Troie n'aura pas lieu, Théâtre complet, op. cit., Acte I, scène V, p. 485.)

7. Pierre Larthomas, Le Langage dramatique, Paris, PUF, 1980, p. 182.

8. Ibid., p. 183. 
autres articles de presse mais surtout en nous intéressant au livre de régie, aux photos de scène et à la correspondance de Louis Jouvet.

La décoration d'Électre fut commandée à un jeune artiste, recommandé par Bérard, Guillaume Monin. Le jeune artiste, s’inspirant de Ledoux ${ }^{9}$, dessine un palais avec des «colonnes majestueuses ${ }^{10}$ et des murs percés de toutes parts. Face au public, trois grandes ouvertures sous des arcades que surplombent des remparts percés eux aussi par trois fois. Côté cour et jardin quatre sorties, deux grandes qui se font face et deux escaliers descendants qui donnent l'impression, comme le rapporte André Frank dans L'Intransigeant, de «s'enfoncer sous la scène ». Jouvet projette d'abord d'équiper les coulisses de cinq tampons (ascenseurs) pour finalement n'en garder que deux. Il installe quatre escaliers descendants et un escalier invisible qui permet d'accéder aux remparts. Ainsi, les personnages «à peine sortis, réapparaîtront sur les remparts $»^{11}$. Ce palais semble tout entier tourné vers le hors-scène, vers ce qui s'est passé « voilà sept ans » et ce qui se passera à la sortie de scène d'Oreste. Ces murs percés invitent le regard à voir au-delà de la scène, et ces escaliers qui s'enfoncent toujours plus bas semblent mener les personnages à la rencontre de leurs destins.

L'éclairage joue aussi un grand rôle. Jouvet commande à la société Gaston Conin pas moins de sept mètres de tubes fluorescents verts et sept mètres de tubes rouges. L'éclairage fut l'une des plus grandes réussites de la pièce. Jouvet joue avec les nuances, les teintes : la lumière éclatante de l'après-midi vire au bleu nuit puis «monte lentement à partir de la scène IV et atteint son maximum d'éclat à la fin de la scène VII, puis va se ternissant progressivement jusqu'au premier récit du Mendiant. À la fin du deuxième récit il se colore de rouge à la cour et au fond », comme l'écrit Marthe Herlin ${ }^{12}$. D'ailleurs, il est intéressant de voir comment à la fin du récit du Mendiant le fond qui préfigure le hors-scène se colore en rouge, comme si le sang versé dans le hors-scène se déversait sur scène pour annoncer cette aurore nouvelle.

Dans le livre de conduite conservé à la Bibliothèque Nationale, et en regard du récit du Mendiant, on trouve ces quelques indications et des schémas des déplacements des comédiens :

À la sortie de scène d'Oreste : «Il sort côté Cour. Électre et Femme Narsès se rapprochent du Mendiant » puis «Femme Narsès fait asseoir Électre ». Pour le Mendiant, le mot d'ordre semble être «mouvement», écrit en rouge plusieurs fois, alors qu'Électre et la Femme Narsès doivent se tenir au centre de la scène, presque immobiles. Dans un des schémas, les déplacements du Men-

9. Claude Nicolas Ledoux, architecte français (1736-1808) est un des précurseurs du néoclassicisme : le pavillon de Mme du Barry, Louveciennes, Barrière de Chartre, Rotonde du parc Monceau...

10. Voir Colette Weil, notice d'Électre, in Jean Giraudoux, Théatre complet, op. cit., p. 1545.

11. Ibid., p. 1546.

12. Id. 
diant semblent correspondre à l'évolution de son récit. Il fait le tour de la scène, du côté jardin au côté cour. Il semble donc faire le tour de ces ouvertures qui encadrent la scène. Son récit comme ses yeux sont tournés vers le hors-scène jusqu'à ce que la fin du récit le ramène au centre de la scène qui se teinte en rouge alors que le cri d'Égisthe retentit accompagné de quelques accords de Vittorio Rieti.

Dans la mise en scène de Pierre Dux à la Comédie-Française en 1959, le palais est percé de grandes ouvertures et est fait de colonnes et de voilages. Ainsi, le passage du hors-scène à la scène s'opère dans un bruissement d'étoffe. En 1999, l'Atelier Théâtre de Lausanne donne une représentation très particulière d'Électre. L'action se déroule dans une piscine, celle où est mort Agamemnon. Le regard du spectateur est invité à plonger dans l'espace concret du meurtre mais aussi dans le hors-scène historique. Tout autour de la piscine des cavités sombres comme autant de portes closes. En effet, le hors-scène n'est plus dans les coulisses, il est là sur la scène et s'étale sur les murs avec un jeu d'ombres qui préfigure, selon les metteurs en scène, «les signes de cauchemar dans la tête d'Électre et dans celle d'Oreste $»^{13}$. Il est intéressent de voir dans cette mise en scène comment le hors-scène intègre l'espace scénique sans disparaître dans sa spécificité puisqu'il n'apparaît ici que comme le reflet du subconscient des personnages et demeure techniquement un espace hors de la scène.

La question du hors-scène est complexe, dans le récit du Mendiant elle est particulièrement problématique. Le texte, qui joue avec délectation de cette ambivalence entre une écriture théâtrale classique et pourtant profondément originale, ne se laisse pas enfermer dans une analyse réductrice. Giraudoux, en véritable dramaturge de l'invisible, nous présente un hors-scène multiple, ambivalent. La notion spatio-temporelle semble un prisme à plusieurs facettes, il est un espace concret et un espace de légende, un temps de la représentation et un temps du mythe... Le hors-scène est le lieu de tous les possibles. La mise en scène se trouve dès lors face au choix d'un axe de mise en scène, d'un superobjectif. Jouvet a opté pour un décor qui ne trace pas de frontières claires entre la scène et le hors-scène mais qui, au contraire, semble avec ses multiples ouvertures devenir comme poreux. Le hors-scène n'est-il pas en fin de compte notre propre mémoire d'hyper lecteur / spectateur qui connaît si bien ses classiques?

13. Interview de Gérard Desarthe in Cabier $n^{\circ} 1$ de l'Atelier Théâtre itinérant. 